\title{
Infrastructural Overview of College Libraries towards Implementation of Virtual Reference Service (VRS): A Study of North-Western India
}

\author{
Subhajit Panda \\ Assistant Librarian, Chandigarh University, \\ Mohali, Punjab, India \\ subhajit.e9641@cumail.in
}

\begin{abstract}
Reference Service enables libraries to provide information to a person who needs it, either directly from an appropriate source, or indirectly by providing the person with the source or teaching him how to find the needed information in the sources. With new technologies, users' information seeking behaviour (ISB) along with their expectations from the reference service has witnessed a sea change, which motivate the Librarians to embrace the Internet as an appropriate medium for information exchange, communication, and instruction, including both synchronous and asynchronous tools and played a pivotal role in transforming the in-person desk-based TRS to VRS. Libraries in India by-and-large have implemented ILMS (e.g. RFID) for their automation but the status of VRS adoption in the Indian context is scarce barring a few cases wherein libraries are providing reference service through email or web-form (asynchronous). Thus, in the light of the above facts the present study has been planned by the researcher as a meaningful understanding and deliberate investigation pertaining to the domain of VRS. The study result reveals that although, VRS was not being offered by almost all of the libraries and Synchronous VRS (SVRS) was offered by none of the libraries under the study. It is clear from the result that the college libraries of North-Western India cater to the need of a heterogeneous user community as the institutions belonging to a wide variety of disciplines, enables the researcher to capture a wide range of perspectives relating to the status of VRS. Again from the study we can infer that the libraries were having basic ICT infrastructure thus making a case for implementing VRS without significant expenditure, which can resolve the shortage of sufficient staff of the libraries in near future.
\end{abstract}

Keywords: Virtual Reference Service, Traditional Reference Service, Information Seeking Behaviour, VRS Implementation, ICT Infrastructure, College Libraries, Librarians' Perspective, North-Western India

\section{Introduction:}

Samuel Green, in his 1876 paper titled, "Personal Relations Between Librarians and Reader," wrote, "helping users locate information is essential because the public is not trained to find information" (Bopp \& Smith, 2001). The assistance of users forms the kernel of reference service the essence of which was beautifully expressed by the father of Library science Dr. S. R. Ranganathan as the process of establishing "contact between the right reader and right book at the right time and in the right personal way" (Ranganathan, 1989). Ranganathan defines Reference Service as: "Personal Service to each reader in helping him to find the documents answering his interest at the moment pin-pointedly, exhaustively and expeditiously" (Ranganathan, 1961). Reference Service enables libraries to meet the information needs of the users (Chowdhury, 2002). In response to developments in society and library use, reference services began in the late 19th and early 20th centuries as the libraries started witnessing an increase in the volume, variety and format of available information resources. 
Assistance to users in finding required information has become more relevant in the 21st century owing to multiplicity, magnitude and enormity of information forms and formats, especially online information.

With new technologies, users' information seeking behaviour (ISB) along with their expectations from the reference service has witnessed a sea change. Users of the current generation now possess a wider range of information needs and inquiries while the sophistication with information searching has also increased tremendously. To meet such information needs and demands, libraries need to expand the scope of reference beyond using the mail, telephone, or fax machine with the help of the computer and the Internet. Technological innovation played a pivotal role in transforming the in-person desk-based traditional reference service (TRS) to VRS.

\section{Literature review:}

Literature reviews are systematic syntheses of previous work around a particular topic. A literature review is a systematic explicit and reproductive method for identifying, evaluating and interpreting the existing body of recorded work produced by researchers, scholars and practitioners (Fink, 1998, p.3). The review of the literature for this study focuses on VRS/DRS as discussed by different authors in their scholarly writings.

Vogus (2020) examined various facets of virtual reference services to reveal the effectiveness of virtual services, how to improve them, and why users prefer one method of virtual reference services to another. Anna \& Srirahayu (2020) evaluated virtual reference and information services at university libraries in Indonesia from two specific aspects: from the aspect of the library that provides virtual reference services and from aspects of users of virtual reference services. The exploratory study of Mawhinney (2020) examined user preferences with regard to virtual reference services and factors that account for these preferences from a different vantage point than previous literature by relying on semi-structured interviews with users. In their article, Mawhinney \& Kochkina (2019) presented the results of a study on virtual reference service, where they seek to determine whether or not the texting service is filling a different information need than chat and email by examining the level of difficulty and the topic of questions using these different methods of communication. Barrett \& Pagotto (2019) examined whether the service model, staffing choices, and policies of its chat reference service were associated with user dissatisfaction, aiming to identify areas where the collaboration is successful and areas which could be improved. In their article chapter Cleveland \& Philbrick (2018) provide an overview of virtual reference services, discuss key issues related to the provision of virtual reference services, review the literature on virtual reference services in academic health sciences libraries, and present a study on the trends in virtual reference services in academic health sciences libraries. Hutchinson (2017) discussed the implementation of LibAnswers by the University of Saskatchewan and its impact on the functioning of the reference service.

\section{Objectives of the Study:}

a) To assess the current status of basic ICT infrastructure \& manpower available at the libraries of North-Western India.

b) To ascertain the current state of reference service being offered in the libraries of North-Western India. 
c) To investigate the availability of instruction based service being offered in the libraries of North-Western India.

d) To investigate the librarians' perception towards traditional and virtual reference service (TRS and VRS) in the libraries of North-Western India.

\section{Scope of the Study:}

The scope of the study comprises various facets pertaining to virtual reference service (VRS) including technological innovations, assessment of their suitability, and current status of reference service in libraries of North-Western India. Various aspects of VRS Web-tool including chat, integrated file sharing, FAQs database, and co-browsing, etc., perceptions of Librarians' regarding the VRS as-well-as, users' awareness and value judgement regarding the same constituted a major study component and provided valuable inputs for devising a realistic framework of VRS implementation and its sustainability.

\section{Research Methodology:}

The research problem investigated by the study was the culmination of several factors including the present infrastructure of actual VRS implementation in north-west Indian libraries, especially SVRS. To gather data for addressing these issues, the quantitative method was found to be appropriate as it facilitates measuring, ranking, categorizing, identifying patterns and making generalizations. The research framework of this survey was designed upon a structured research questionnaire created through Google form and circulated online via email to the eminent college library professionals of north-western India. The questionnaire was prepared for library professionals with a view to understand, describe, interpret, contextualize, and gain in-depth insight pertaining to the research problem and research questions/objectives of the present study from their perspective. It took almost 8 months (from March 2020 to October 2020) and distributed among 350 library professionals of which $302(86 \%)$ took part in this survey from different region of north-western India.

\begin{tabular}{|c|l|c|c|}
\hline SN & \multicolumn{1}{|c|}{ State } & Respondent & Non-Respondent \\
\hline 1 & Ladakh & 2 & 2 \\
\hline 2 & Jammu \& Kashmir & 33 & 7 \\
\hline 3 & Himachal Pradesh & 21 & 12 \\
\hline 4 & Punjab & 53 & 3 \\
\hline 5 & Haryana & 48 & 23 \\
\hline 6 & Rajasthan & $\mathbf{3 0 2}(\mathbf{8 6 \%})$ & $\mathbf{4 8}(\mathbf{1 4 \%})$ \\
\hline \multicolumn{2}{|l|}{ Total } & $\mathbf{3 5 0}(\mathbf{1 0 0 \% )}$ \\
\hline All Total
\end{tabular}

Table 1: State wise Cluster of Sample Universe 


\section{Data Analysis:}

Data analysis and interpretation holds a crucial position in the research process. After collection of data it was imported as .csv file (Google sheet) for further analysis and visualization using spreadsheet software. Finally the data analysis for the present research was done quantitatively with the help of both descriptive statistics and inferential statistics. It aims to organize, classify and summarize the data being collected for better comprehension and interpretation leading to understand and explore answers or solutions to the research problem which originally triggered the research.

\section{Results and Discussion:}

The respondents of the varied disciplines may exhibit a wide range of attributes, behaviours, experiences, incidents, qualities, situations, and so forth which facilitates gaining greater insights understanding the variation in librarians' perspectives, ranging from Current Status of Reference Service, reference collection size, VRS infrastructure and librarians' perception regarding VRS.

\subsection{Core Disciplines of the Sample institutions:}

The universe of the study comprised selected college libraries of the north-western India. Library professionals from those institutions formed the set of respondents. The general information about the core discipline of those libraries are discussed bellow.

\begin{tabular}{|c|l|c|c|}
\hline SN & \multicolumn{1}{|c|}{ Core Discipline } & No & Percentage \\
\hline 1 & Science & 21 & 7 \\
\hline 2 & Social Science & 59 & 20 \\
\hline 3 & Engineering \& Tech. & 21 & 7 \\
\hline 4 & Health \& medicine & 9 & 3 \\
\hline 5 & Engineering \& Tech. \& Health and medicine & 15 & 5 \\
\hline 6 & $\begin{array}{l}\text { Science, Engineering \& Tech and Health \& } \\
\text { medicine }\end{array}$ & 29 & 10 \\
\hline 7 & General (Multidisciplinary) & 137 & 45 \\
\hline 8 & Business \& I.T Total & $\mathbf{3 0 2}$ & $\mathbf{1 0 0}$ \\
\hline
\end{tabular}

Table 2: Core disciplines of sample institutions

Table 2 indicates that there are all total 8 types of core discipline among the sample institutions from "Science" to "Engineering \& Tech.", "Health \& medicine" to "Business \& I.T." etc. Among them almost half $(=45 \%, 137)$ libraries belongs to "General" or multidisciplinary field while "Social 
Science" libraries positioned second with $20 \%(=59)$ coverage. It is clear from the above observations that the libraries of the above institutions cater to the need of a heterogeneous user community belonging to a wide variety of disciplines. This enables the researcher to capture a wide range of perspectives relating to the status of Virtual Reference Service (VRS).

\subsection{Availability of Human Resources:}

For effective implementation of VRS in a library, availability of efficient library staff is one of the essential needs. Following table give detailed overview of number of staff in the sample libraries according to their respective designation.

\begin{tabular}{|c|l|c|c|}
\hline SN & \multicolumn{1}{|c|}{ Designation } & No & $\begin{array}{c}\text { Staff Per } \\
\text { Institution }\end{array}$ \\
\hline 1 & Librarian & 227 & 0.8 \\
\hline 2 & Assistant Librarian & 81 & 0.3 \\
\hline 3 & Library Assistant & 138 & 0.5 \\
\hline 4 & Para-Professional & 22 & 0.1 \\
\hline 5 & Library Attendant & 258 & 0.9 \\
\hline 6 & Clerical Staff & 302 & 1.0 \\
\hline
\end{tabular}

Table 3: Number of staff in the library

Table 3 above describes the availability of library professionals and other staff in the select institutions' libraries. It was noted that almost 44 libraries had no 'librarian' and 221 libraries either didn't have any 'Assistant Librarian' or the position. Moreover, half of the library $(=138)$ occupied with 'Library Assistant' and almost 90\% (=258) library have a 'Library Attended'. For every library, only the 'Clerical Staff' availability was verified. Though the upper study result revealed that the sample libraries are not occupied with sufficient library staffs, a fewer staff can manage VRS efficiently and systematically with its facility to allow librarians to take up more queries in less time.

\subsection{Availability of infrastructure and efficient manpower:}

Availability of ICT infrastructure and trained staffs are conducive for Computer-Mediated Environment (CME) which is essential for providing Virtual Reference Service (VRS). The following table scrutinized about the availability of existing ICT infrastructure and trained staffs in sample libraries. 


\begin{tabular}{|c|c|c|c|c|c|}
\hline & $\begin{array}{c}\text { Highly } \\
\text { Inadequate } \\
\text { n(\%) }\end{array}$ & $\begin{array}{c}\text { Inadequate } \\
\mathbf{n}(\%)\end{array}$ & $\begin{array}{c}\text { Adequate } \\
\text { n(\%) }\end{array}$ & $\begin{array}{c}\text { Highly } \\
\text { Adequate } \\
\text { /Suitable } \\
\text { n(\%) }\end{array}$ & $\begin{array}{l}\text { Total } \\
\mathbf{n}(\%)\end{array}$ \\
\hline Computer & $\begin{array}{c}0 \\
(0)\end{array}$ & $\begin{array}{l}16 \\
(5)\end{array}$ & $\begin{array}{c}95 \\
(32)\end{array}$ & $\begin{array}{l}191 \\
(63)\end{array}$ & $\begin{array}{c}302 \\
(100)\end{array}$ \\
\hline Server & $\begin{array}{c}6 \\
(2)\end{array}$ & $\begin{array}{c}36 \\
(12)\end{array}$ & $\begin{array}{l}133 \\
(44)\end{array}$ & $\begin{array}{l}127 \\
(42)\end{array}$ & $\begin{array}{c}302 \\
(100)\end{array}$ \\
\hline $\begin{array}{l}\text { Library } \\
\text { automation } \\
\text { software }\end{array}$ & $\begin{array}{c}0 \\
(0)\end{array}$ & $\begin{array}{c}33 \\
(11)\end{array}$ & $\begin{array}{l}69 \\
(23)\end{array}$ & $\begin{array}{l}199 \\
(66)\end{array}$ & $\begin{array}{c}302 \\
(100)\end{array}$ \\
\hline Official cell phone & $\begin{array}{c}45 \\
(15)\end{array}$ & $\begin{array}{l}21 \\
(7)\end{array}$ & $\begin{array}{l}202 \\
(67)\end{array}$ & $\begin{array}{c}34 \\
(11)\end{array}$ & $\begin{array}{c}302 \\
(100)\end{array}$ \\
\hline ICT Trained staff & $\begin{array}{c}0 \\
(0)\end{array}$ & $\begin{array}{c}30 \\
(10)\end{array}$ & $\begin{array}{l}142 \\
(47)\end{array}$ & $\begin{array}{l}130 \\
(43)\end{array}$ & $\begin{array}{c}302 \\
(100)\end{array}$ \\
\hline $\begin{array}{l}\text { Internet } \\
\text { connection }\end{array}$ & $\begin{array}{c}0 \\
(0)\end{array}$ & $\begin{array}{c}0 \\
(0)\end{array}$ & $\begin{array}{l}159 \\
(53)\end{array}$ & $\begin{array}{l}143 \\
(47)\end{array}$ & $\begin{array}{c}302 \\
(100)\end{array}$ \\
\hline $\begin{array}{l}\text { Local Area } \\
\text { Network (LAN) }\end{array}$ & $\begin{array}{c}0 \\
(0)\end{array}$ & $\begin{array}{c}0 \\
(0)\end{array}$ & $\begin{array}{l}124 \\
(41)\end{array}$ & $\begin{array}{l}178 \\
(59)\end{array}$ & $\begin{array}{c}302 \\
(100)\end{array}$ \\
\hline Wi-Fi & $\begin{array}{l}15 \\
(5)\end{array}$ & $\begin{array}{c}0 \\
(0)\end{array}$ & $\begin{array}{l}129 \\
(43)\end{array}$ & $\begin{array}{l}158 \\
(52)\end{array}$ & $\begin{array}{c}302 \\
(100)\end{array}$ \\
\hline Scanner & $\begin{array}{c}0 \\
(0)\end{array}$ & $\begin{array}{l}12 \\
(4)\end{array}$ & $\begin{array}{l}145 \\
(48)\end{array}$ & $\begin{array}{l}145 \\
(48)\end{array}$ & $\begin{array}{c}302 \\
(100)\end{array}$ \\
\hline Printer & $\begin{array}{c}0 \\
(0)\end{array}$ & $\begin{array}{l}0 \\
(0)\end{array}$ & $\begin{array}{l}157 \\
(52)\end{array}$ & $\begin{array}{l}145 \\
(48)\end{array}$ & $\begin{array}{c}302 \\
(100)\end{array}$ \\
\hline Library web site & $\begin{array}{l}14 \\
(5)\end{array}$ & $\begin{array}{l}16 \\
(5)\end{array}$ & $\begin{array}{l}128 \\
(42)\end{array}$ & $\begin{array}{l}144 \\
(48)\end{array}$ & $\begin{array}{c}302 \\
(100)\end{array}$ \\
\hline OPAC & $\begin{array}{c}32 \\
(11)\end{array}$ & $\begin{array}{l}24 \\
(8)\end{array}$ & $\begin{array}{l}134 \\
(44)\end{array}$ & $\begin{array}{l}112 \\
(37)\end{array}$ & $\begin{array}{c}302 \\
(100)\end{array}$ \\
\hline $\begin{array}{l}\text { VRS } \\
\text { Software/Tool }\end{array}$ & $\begin{array}{c}94 \\
(31)\end{array}$ & $\begin{array}{l}121 \\
(40)\end{array}$ & $\begin{array}{c}68 \\
(23)\end{array}$ & $\begin{array}{l}19 \\
(6)\end{array}$ & $\begin{array}{c}302 \\
(100)\end{array}$ \\
\hline
\end{tabular}

Table 4: Existing infrastructure and manpower available in the library for VRS

Table 4 indicates that little more than $50 \%$ of the libraries found 'computer', 'library automation software', 'LAN' \& 'Wi-Fi' highly adequate to serve their patron while little more than forty percent mentioned the adequacy of this infrastructure. It was also found that 'Official cell phone' (78\%), 'ICT Trained staff' (90\%), 'Internet connection' (100\%), 'Local Area Network (LAN)' (100\%), 'Scanner' (96\%), 'Printer' (100\%), 'Library web site' (90\%), 'OPAC' (81\%) were available (highly adequate and adequate) in almost all the libraries. The table also reveals that only in some of the library 'Library automation software', 'Wi-Fi', 'library website' and 'OPAC' were highly inadequate. It was found from the above table that most of the libraries were having basic ICT infrastructure thus making a case for implementing VRS, (both synchronous and asynchronous) including chat-based reference service. Thus libraries can plan for taking VRS initiatives without significant expenditure towards building ICT infrastructure. 


\subsection{Types of Reference and Information services provided in the library}

A question was asked to the librarians of select institutions regarding types of Reference and Information Services (RISS) provided in their respective libraries. As per the responses received the data was tabulated.

\begin{tabular}{|c|c|c|c|c|c|c|}
\hline & $\begin{array}{c}\text { Very } \\
\text { Frequently } \\
\text { n(\%) }\end{array}$ & $\begin{array}{l}\text { Frequently } \\
\text { n(\%) }\end{array}$ & $\begin{array}{c}\text { Sometimes/ } \\
\text { occasionally } \\
\text { n }(\%)\end{array}$ & $\begin{array}{l}\text { Rarely } \\
\mathbf{n}(\%)\end{array}$ & $\begin{array}{l}\text { Never } \\
\text { n(\%) }\end{array}$ & Total \\
\hline $\begin{array}{l}\text { Quick/Ready } \\
\text { reference } \\
\text { and other } \\
\text { directional } \\
\text { services }\end{array}$ & $\begin{array}{c}97 \\
(32)\end{array}$ & $\begin{array}{l}142 \\
(47)\end{array}$ & $\begin{array}{c}30 \\
(10)\end{array}$ & $\begin{array}{c}33 \\
(11)\end{array}$ & $\begin{array}{c}0 \\
(0)\end{array}$ & $\begin{array}{c}302 \\
(100)\end{array}$ \\
\hline $\begin{array}{l}\text { Information } \\
\text { and Referral } \\
\text { services }\end{array}$ & $\begin{array}{l}48 \\
(16)\end{array}$ & $\begin{array}{l}181 \\
(60)\end{array}$ & $\begin{array}{c}66 \\
(22)\end{array}$ & $\begin{array}{c}7 \\
(2)\end{array}$ & $\begin{array}{c}0 \\
(0)\end{array}$ & $\begin{array}{c}302 \\
(100)\end{array}$ \\
\hline $\begin{array}{l}\text { Inter Library } \\
\text { Loan }\end{array}$ & $\begin{array}{c}36 \\
(12)\end{array}$ & $\begin{array}{l}133 \\
(44)\end{array}$ & $\begin{array}{c}33 \\
(11)\end{array}$ & $\begin{array}{l}100 \\
(33)\end{array}$ & $\begin{array}{c}0 \\
(0)\end{array}$ & $\begin{array}{c}302 \\
(100)\end{array}$ \\
\hline $\begin{array}{l}\text { Document } \\
\text { Delivery }\end{array}$ & $\begin{array}{l}82 \\
(27)\end{array}$ & $\begin{array}{c}81 \\
(27)\end{array}$ & $\begin{array}{c}63 \\
(21)\end{array}$ & $\begin{array}{c}54 \\
(18)\end{array}$ & $\begin{array}{l}22 \\
(7)\end{array}$ & $\begin{array}{c}302 \\
(100)\end{array}$ \\
\hline $\begin{array}{l}\text { Fee- based } \\
\text { services }\end{array}$ & $\begin{array}{c}0 \\
(0)\end{array}$ & $\begin{array}{c}6 \\
(2)\end{array}$ & $\begin{array}{c}45 \\
(15)\end{array}$ & $\begin{array}{l}24 \\
(8)\end{array}$ & $\begin{array}{l}227 \\
(75)\end{array}$ & $\begin{array}{c}302 \\
(100)\end{array}$ \\
\hline Procedural & $\begin{array}{c}0 \\
(0)\end{array}$ & $\begin{array}{c}51 \\
(17)\end{array}$ & $\begin{array}{c}66 \\
(22)\end{array}$ & $\begin{array}{l}67 \\
(22)\end{array}$ & $\begin{array}{l}118 \\
(39)\end{array}$ & $\begin{array}{c}302 \\
(100)\end{array}$ \\
\hline $\begin{array}{l}\text { Long Range } \\
\text { Reference } \\
\text { Service }\end{array}$ & $\begin{array}{l}38 \\
(13)\end{array}$ & $\begin{array}{c}40 \\
(13)\end{array}$ & $\begin{array}{l}115 \\
(38)\end{array}$ & $\begin{array}{l}88 \\
(29)\end{array}$ & $\begin{array}{c}7 \\
(21)\end{array}$ & $\begin{array}{c}302 \\
(100)\end{array}$ \\
\hline E-mail alerts & $\begin{array}{c}79 \\
(26)\end{array}$ & $\begin{array}{l}127 \\
(42)\end{array}$ & $\begin{array}{c}36 \\
(12)\end{array}$ & $\begin{array}{c}60 \\
(20)\end{array}$ & $\begin{array}{c}0 \\
(0)\end{array}$ & $\begin{array}{c}302 \\
(100)\end{array}$ \\
\hline $\begin{array}{l}\text { News paper } \\
\text { clipping }\end{array}$ & $\begin{array}{l}78 \\
(26)\end{array}$ & $\begin{array}{l}111 \\
(37)\end{array}$ & $\begin{array}{c}63 \\
(21)\end{array}$ & $\begin{array}{c}34 \\
(11)\end{array}$ & $\begin{array}{l}16 \\
(5)\end{array}$ & $\begin{array}{c}302 \\
(100)\end{array}$ \\
\hline $\begin{array}{l}\text { Electronic } \\
\text { clipping }\end{array}$ & $\begin{array}{c}37 \\
(12)\end{array}$ & $\begin{array}{c}94 \\
(31)\end{array}$ & $\begin{array}{c}57 \\
(19)\end{array}$ & $\begin{array}{l}11 \\
(4)\end{array}$ & $\begin{array}{l}103 \\
(34)\end{array}$ & $\begin{array}{c}302 \\
(100)\end{array}$ \\
\hline $\begin{array}{l}\text { Indexing and } \\
\text { abstracting }\end{array}$ & $\begin{array}{c}51 \\
(17)\end{array}$ & $\begin{array}{c}48 \\
(16)\end{array}$ & $\begin{array}{c}43 \\
(14)\end{array}$ & $\begin{array}{c}48 \\
(16)\end{array}$ & $\begin{array}{l}112 \\
(37)\end{array}$ & $\begin{array}{c}302 \\
(100)\end{array}$ \\
\hline SDI & $\begin{array}{c}94 \\
(31)\end{array}$ & $\begin{array}{l}42 \\
(14)\end{array}$ & $\begin{array}{l}45 \\
(15)\end{array}$ & $\begin{array}{c}30 \\
(10)\end{array}$ & $\begin{array}{c}91 \\
(30)\end{array}$ & $\begin{array}{c}302 \\
(100)\end{array}$ \\
\hline $\begin{array}{l}\text { Display of } \\
\text { new arrivals }\end{array}$ & $\begin{array}{l}127 \\
(42)\end{array}$ & $\begin{array}{l}160 \\
(53)\end{array}$ & $\begin{array}{l}15 \\
(5)\end{array}$ & $\begin{array}{c}0 \\
(0)\end{array}$ & $\begin{array}{c}0 \\
(0)\end{array}$ & $\begin{array}{c}302 \\
(100)\end{array}$ \\
\hline
\end{tabular}

Table 5: Types of Reference and Information services provided in the library

Table 5 makes it clear that the highest response $(60 \%)$ for "information and referral services" was provided 'Frequently' while $16 \%$ provided this service 'very frequently'. 'Display of new arrivals' (95\%) and 'quick/ready reference and other directional services' (79\%) were provided regularly ('Very Frequently' and 'Frequently') by the majority of the institutional libraries. 'E-mail alerts' (68\%) and 'news paper clipping' (63\%) were regularly (frequently and very frequently) provided by the libraries. 'Inter-Library Loan' (57\%) and 'SDI' (45\%) were also regularly provided by a little less than $60 \%$ and little less than half of the libraries respectively. About $75 \%$ librarian opined that they never facilitated 'Fee-based services' in their libraries while 39\% libraries never offered 'Procedural' and 21\% 'Long Range Reference Service' to their patron. The result clearly indicates that the sample 
libraries were providing either traditional desk-based in-person reference service or by asynchronous mode of VRS like Email. So, there exists a great opportunity for those libraries to initiate VRS at their campuses.

\subsection{Instruction based services provided by the library:}

Library instruction also referred to as user education, library orientation or information literacy is a process of making library patrons understand how to make effective and efficient use of library resources and information through the acquisition of skills to identify, locate, search, retrieve and exploit information in the library (Igbena, 1990). Instruction based services are very useful services assisting the users in searching, locating, finding, evaluating and using information with least effort. This facilitates better use of library resources, as they can be put to maximum utilization.

\begin{tabular}{|c|c|c|c|c|c|c|}
\hline & $\begin{array}{c}\text { Very } \\
\text { Frequently } \\
\text { n(\%) }\end{array}$ & $\begin{array}{l}\text { Frequently } \\
\text { n(\%) }\end{array}$ & $\begin{array}{c}\text { Sometimes/ } \\
\text { occasionally } \\
\text { n }(\%)\end{array}$ & $\begin{array}{l}\text { Rarely } \\
\mathbf{n}(\%)\end{array}$ & $\begin{array}{l}\text { Never } \\
\text { n(\%) }\end{array}$ & $\begin{array}{l}\text { Total } \\
\text { n(\%) }\end{array}$ \\
\hline $\begin{array}{l}\text { One to one } \\
\text { instruction }\end{array}$ & $\begin{array}{l}112 \\
(37)\end{array}$ & $\begin{array}{c}33 \\
(11)\end{array}$ & $\begin{array}{l}109 \\
(36)\end{array}$ & $\begin{array}{c}32 \\
(11)\end{array}$ & $\begin{array}{l}16 \\
(5)\end{array}$ & $\begin{array}{c}302 \\
(100)\end{array}$ \\
\hline $\begin{array}{l}\text { Group } \\
\text { instruction }\end{array}$ & $\begin{array}{c}30 \\
(10)\end{array}$ & $\begin{array}{l}109 \\
(36)\end{array}$ & $\begin{array}{c}82 \\
(27)\end{array}$ & $\begin{array}{l}17 \\
(6)\end{array}$ & $\begin{array}{c}64 \\
(21)\end{array}$ & $\begin{array}{c}302 \\
(100)\end{array}$ \\
\hline $\begin{array}{l}\text { Orientation } \\
\text { tours }\end{array}$ & $\begin{array}{l}11 \\
(4)\end{array}$ & $\begin{array}{l}145 \\
(48)\end{array}$ & $\begin{array}{l}118 \\
(39)\end{array}$ & $\begin{array}{l}21 \\
(7)\end{array}$ & $\begin{array}{l}7 \\
(2)\end{array}$ & $\begin{array}{c}302 \\
(100)\end{array}$ \\
\hline $\begin{array}{l}\text { Induction } \\
\text { sessions }\end{array}$ & $\begin{array}{l}24 \\
(8)\end{array}$ & $\begin{array}{c}40 \\
(13)\end{array}$ & $\begin{array}{c}63 \\
(21)\end{array}$ & $\begin{array}{c}63 \\
(21)\end{array}$ & $\begin{array}{l}112 \\
(37)\end{array}$ & $\begin{array}{c}302 \\
(100)\end{array}$ \\
\hline $\begin{array}{l}\text { Information } \\
\text { literacy } \\
\text { sessions }\end{array}$ & $\begin{array}{c}32 \\
(11)\end{array}$ & $\begin{array}{c}46 \\
(15)\end{array}$ & $\begin{array}{c}79 \\
(26)\end{array}$ & $\begin{array}{l}66 \\
(22)\end{array}$ & $\begin{array}{c}79 \\
(26)\end{array}$ & $\begin{array}{c}302 \\
(100)\end{array}$ \\
\hline $\begin{array}{l}\text { Printed } \\
\text { guides and } \\
\text { handouts }\end{array}$ & $\begin{array}{c}31 \\
(10)\end{array}$ & $\begin{array}{l}99 \\
(33)\end{array}$ & $\begin{array}{c}61 \\
(20)\end{array}$ & $\begin{array}{l}15 \\
(5)\end{array}$ & $\begin{array}{c}96 \\
(32)\end{array}$ & $\begin{array}{c}302 \\
(100)\end{array}$ \\
\hline $\begin{array}{l}\text { Audiovisual } \\
\text { presentations }\end{array}$ & $\begin{array}{c}36 \\
(12)\end{array}$ & $\begin{array}{c}39 \\
(13)\end{array}$ & $\begin{array}{l}154 \\
(51)\end{array}$ & $\begin{array}{l}24 \\
(8)\end{array}$ & $\begin{array}{c}49 \\
(16)\end{array}$ & $\begin{array}{c}302 \\
(100)\end{array}$ \\
\hline $\begin{array}{l}\text { Web-based } \\
\text { guides and } \\
\text { handouts }\end{array}$ & $\begin{array}{l}57 \\
(19)\end{array}$ & $\begin{array}{c}64 \\
(21)\end{array}$ & $\begin{array}{l}30 \\
(10)\end{array}$ & $\begin{array}{c}9 \\
(3)\end{array}$ & $\begin{array}{l}142 \\
(47)\end{array}$ & $\begin{array}{c}302 \\
(100)\end{array}$ \\
\hline $\begin{array}{l}\text { Course-integr } \\
\text { ated } \\
\text { instructions }\end{array}$ & $\begin{array}{c}0 \\
(0)\end{array}$ & $\begin{array}{c}0 \\
(0)\end{array}$ & $\begin{array}{c}97 \\
(32)\end{array}$ & $\begin{array}{l}133 \\
(44)\end{array}$ & $\begin{array}{l}72 \\
(24)\end{array}$ & $\begin{array}{c}302 \\
(100)\end{array}$ \\
\hline $\begin{array}{l}\text { Database/e-re } \\
\text { sources } \\
\text { Mediated } \\
\text { searching }\end{array}$ & $\begin{array}{l}79 \\
(26)\end{array}$ & $\begin{array}{l}159 \\
(53)\end{array}$ & $\begin{array}{l}16 \\
(5)\end{array}$ & $\begin{array}{l}15 \\
(5)\end{array}$ & $\begin{array}{l}33 \\
(11)\end{array}$ & $\begin{array}{c}302 \\
(100)\end{array}$ \\
\hline $\begin{array}{l}\text { Access/search } \\
\text { ing OPAC }\end{array}$ & $\begin{array}{l}157 \\
(52)\end{array}$ & $\begin{array}{c}94 \\
(31)\end{array}$ & $\begin{array}{l}27 \\
(9)\end{array}$ & $\begin{array}{c}0 \\
(0)\end{array}$ & $\begin{array}{l}24 \\
(8)\end{array}$ & $\begin{array}{c}302 \\
(100)\end{array}$ \\
\hline
\end{tabular}

Table 6: Instruction based services provided by the library

Table 6 reveals the preference of instruction based services provided by the libraries of select sample institutions. It was found that the highest response (52\%) for 'access or searching OPAC' was in 'very 
frequently' provided category while $31 \%$ provided this service 'frequently'. Little more than fifty percent $(53 \%)$ of the librarians opined 'database/e-resources mediated searching' were provided frequently while $26 \%$ responded that they provided this service very frequently, followed by 'one to one instruction' (37\%). 'Orientation tour' (52\%) and 'group instruction' (46\%) offered by the select libraries occupied Rank 4 \& 5 respectively. Other instructions based services provided by select institution libraries were 'audiovisual presentation', 'printed guides and handouts', 'web-based guides and handouts', 'information literacy sessions' and 'course-integrated instructions' ranging from R6 to R11 with no sharp difference. The table/result reveals that all the libraries under study were providing instruction based services by and large.

\subsection{Librarians' perception regarding various types of reference service:}

\begin{tabular}{|l|c|c|c|c|}
\hline & $\begin{array}{c}\text { Traditional } \\
\text { reference Desk } \\
\mathbf{n ( \% )}\end{array}$ & $\begin{array}{c}\text { VRS } \\
\mathbf{n ( \% )}\end{array}$ & $\begin{array}{c}\text { No } \\
\text { Impact } \\
\mathbf{n}(\%)\end{array}$ & $\begin{array}{c}\text { Total } \\
\mathbf{n}(\%)\end{array}$ \\
\hline $\begin{array}{l}\text { Quick/Ready reference } \\
\text { questions }\end{array}$ & 63 & 160 & 79 & 302 \\
$(21)$ & $(53)$ & $(26)$ & $(100)$ \\
\hline $\begin{array}{l}\text { Detailed, research } \\
\text { questions }\end{array}$ & 96 & 126 & 80 & 302 \\
\hline $\begin{array}{l}\text { Reader's advisory } \\
\text { questions }\end{array}$ & $(32)$ & $(42)$ & $(26)$ & $(100)$ \\
\hline Directional questions & $(53)$ & $(21)$ & $(26)$ & $(100)$ \\
\hline Procedural questions & 112 & 127 & 63 & 302 \\
& $(37)$ & $(42)$ & $(21)$ & $(100)$ \\
\hline
\end{tabular}

Table 7: Perception regarding various types of reference service

Table 7 depicts librarians' perception regarding the preference of TRS over VRS and vice versa. It indicates that more than $50 \%$ librarians pointed out that 'quick/ready reference questions' and 'procedural questions' can be better handled by VRS than TRS. As per the librarians of $42 \%$ institutional libraries, 'detailed research questions' as well as 'directional questions' are more effectively answered through VRS than TRS. However, in the case of 'readers advisory questions' librarians of $53 \%(=160)$ institutions opined that TRS would be a more effective method than VRS. This perception comes along with the belief that VRS can play a major role and add value to the existing reference Service model. 


\section{Conclusion:}

In the current e-centric world and changing user information needs and information-seeking behaviour (ISB), the demand for online information services is growing day by day. The biggest issue is how the libraries are responding to this paradigm shift. It has become imperative to critically assess the perception, perspective and attitudes of the library and information professionals and users regarding VRS. The study has attempted to explore and demonstrate the function of free VRS tools and services with pointing the perception of college librarians of north-western India for the same. And the study result suggests that the libraries should initiate VRS to extend the scope of Traditional Reference Service (TRS) in terms of time and space to make users more successful and productive. And to do this, the librarians should acquire essential competencies and skills pertaining to ICT driven communication. Upon the introduction of VRS, the user training program, proper implementation \& revision of policy \& guidelines, the procurement of the new communication technologies \& other tools, the initiation of a collective or cooperative VRS movement to break through budgeted constraints and proper marketing to conscious users may be a potential solution. Through innovative and instant communication tools, used in VRS, the reference librarians portray or showcase the value and worth of the library among the users. Users can only realise the benefits and important of synchronous mode of VRS when the libraries take initiative in this direction.

\section{References:}

Anna, N., \& Srirahayu, D. (2020). Evaluation of Virtual Reference and Information Services at University Libraries in Indonesia. Library Philosophy and Practice (E-Journal), https://digitalcommons.unl.edu/libphilprac/3583/

Barrett, K., \& Pagotto, S. (2019). Local Users, Consortial Providers: Seeking Points of Dissatisfaction with a Collaborative Virtual Reference Service. Evidence Based Library and Information Practice, 14(4), 2-20. https://doi.org/10.18438/eblip29624

Bopp, R. E., \& Smith, L. C. (2001). Reference and information services: an introduction. Libraries Unlimited, Englewood, Colo.

Chowdhury, G. G. (2002). Digital libraries and reference services: present and future. Journal of Documentation, 58(3), 258-283. https://doi.org/10.1108/00220410210425809

Cleveland, A. D., \& Philbrick, J. L. (2018). Virtual Reference Services for the Academic Health Sciences Librarian 2.0. In Medical Librarian 2.0: Use of Web 2.0 Technologies in Reference Services (pp. 25-49). The Haworth Press, Inc. https://doi.org/10.4324/9780203823385-3

Fink, A. G. (1998). Conducting research literature reviews: From paper to the Internet (2nd ed.). Thousand Oaks: Sage Publications.

Green, S. S. (1876). Personal relations between librarians and readers. Library Journal, 1(October), 74-81.

Hutchinson, T. (2017). Using LibAnswers in the Archives: A Review and Implementation Report. Practical Technology for Archives, 1(7). https://hdl.handle.net/1813/76862

Igbena, F. A. (1990). User education: A comparative analysis of UNN and Anambra State College of Education, Awka, unpublished BLS Project, University of Nigeria, Nsukka.

Mawhinney, T. (2020). User preferences related to virtual reference services in an academic library. The Journal of Academic Librarianship, 46(1), 102094. https://doi.org/10.1016/j.acalib.2019.102094 
Mawhinney, T., \& Kochkina, S. (2019). Is the Medium the Message? Examining Transactions Conducted via Text in Comparison with Traditional Virtual Reference Methods. Journal of Library \& Information Services in Distance Learning, 13(1-2), 56-73. https://doi.org/10.1080/1533290x.2018.1499236

Ranganathan, S. R. (1961). Reference service (2nd ed.). Asia Publishing House, Sarada Ranganathan Endowment for Library Science, Series 8.

Ranganathan, S. R. (1989). Philosophy of library classification. Sarada Ranganathan Endowment for Library Science; Bangalore.

Vogus, B. (2020). Examining virtual reference services in academic libraries. Public Services Quarterly, 16(4), 249-253. https://doi.org/10.1080/15228959.2020.1818664

\section{Further Reading:}

Lauer, J. D., \& McKinzie, S. (2002). Bad Moon Rising. The Reference Librarian, 38(79-80), 45-56. https://doi.org/10.1300/j120v38n79_04

McClennen, M., \& Memmott, P. (2001). Roles in digital reference. Information Technology and Libraries, 20(3), 143-148. http://search.pro-quest.com.proxy-library.ashford.edu/docview/2158 30595?accountid=32521

Shakeri, S., Akbaridaryan, S., \& Mohammadi, F. (2012). Comparison of Traditional and Digital Reference Questions and Responses at the National Library and Archives of I.R. of Iran (NLAI). Reference \& User Services Quarterly, 52(2), 136-144. https://doi.org/10.5860/rusq.52n2.136

Sharma, S., Ashok Kumar, A. K., \& Singh, M. (2004). Digital Reference Service. DESIDOC Bulletin of Information Technology, 24(6), 11-18. https://doi.org/10.14429/dbit.24.6.3641

Zanin-Yost, A. (2004). Digital reference: What the past has taught us and what the future will hold Library Philosophy \& Practice, 7(1), 1-16. 